\title{
Correlation between coronary artery disease and obstructive sleep apnea syndrome and analysis of risk factors
}

\author{
JIDONG ZHANG $^{1 *}$, YU SONG ${ }^{2 *}$, YANG JI ${ }^{1}$, YIYING SONG ${ }^{3}$, \\ SHANGLANG CAI ${ }^{1}$, YANLING YU ${ }^{2}$, SONG LIU ${ }^{1}$ and WENZHONG ZHANG ${ }^{1}$ \\ ${ }^{1}$ Department of Cardiology, The Affiliated Hospital of Medical College Qingdao University; \\ ${ }^{2}$ Department of Orthodontics, Qingdao Stomatological Hospital; ${ }^{3}$ Department of Invasive Technology, \\ The Affiliated Hospital of Medical College Qingdao University, Qingdao, Shandong 266001, P.R. China
}

Received November 3, 2017; Accepted March 9, 2018

DOI: $10.3892 /$ etm.2018.6070

\begin{abstract}
The correlation between coronary artery disease (CAD) and obstructive sleep apnea syndrome (OSAS) was investigated to analyze its risk factors. A total of 84 patients with suspected CAD due to chest tightness and pain or nocturnal angina, were selected. They were admitted and received coronary angiography in The Affiliated Hospital of Medical College Qingdao University from March, 2016 to June, 2017. The vital signs were monitored, and the sleep monitoring was performed before and after coronary angiography. Before angiography, the fasting blood was drawn for blood biochemical detection, followed by routine electrocardiogram and echocardiographic examination. In addition, the body mass index was calculated and whether patients suffered from hypertension and diabetes mellitus was observed. The patients were divided into the control group (patients with a negative coronary angiography) and the CAD group (patients with a positive coronary angiography). There were 34 cases in the control group, including 21 cases of OSAS $(61.76 \%)$, and 50 in the CAD group, including 40 cases of OSAS $(80.00 \%)$. Statistical analysis revealed that there were statistically significant differences in the apnea hypopnea index (AHI), lowest oxygen saturation, degree of coronary stenosis (Gensini score) and triglyceride level between the two groups $(\mathrm{P}<0.05)$. There were no statistically significant differences in the cholesterol level and prevalence rates of hypertension and diabetes mellitus between the two groups. Logistic regression analysis revealed that smoking and AHI $>20$ were the risk factors of CAD (OR=7.036 and 5.377). Thus, CAD is closely
\end{abstract}

Correspondence to: Dr Wenzhong Zhang, Department of Cardiology, The Affiliated Hospital of Medical College Qingdao University, 16 Jiangsu Road, Qingdao, Shandong 266001, P.R. China E-mail: xxmczwz@163.com

*Contributed equally

Key words: coronary artery disease, sleep apnea, sleep respiratory monitoring, correlation, risk factors correlated with OSAS and AHI $>20$ is one of the risk factors of CAD.

\section{Introduction}

Sleep apnea syndrome (SAS) is a sleep respiratory disturbance disease, which is characterized by a high incidence rate, many complications and potential risks (1). Obstructive sleep apnea syndrome (OSAS) accounts for the majority of cases clinically. Epidemiological survey showed that the incidence rate of OSAS is $2-4 \%$ in adults, $4.63 \%$ in individuals aged $>30$ years and up to $30-50 \%$ in individuals complicated with hypertension and coronary artery disease (CAD) $(2,3)$. OSAS is defined as follows: Repeated attack of apnea and hypopnea for more than 30 times during the $7 \mathrm{~h}$ sleep at night, or $\mathrm{AHI} \geq 5$. Polysomnography (PSG) detection is the gold standard of OSAS (4). OSAS can manifest in multiple organ system damage, leading to functional and organic changes in vital organs $(5,6)$. Sleep structural disorder and hypoxemia in sleep stage are important features of OSAS. The repeated hypoxemia and hypercapnia during sleep result in a series of pathophysiological changes in the body, which can promote the development of CAD, and increase the morbidity and mortality rates of CAD and other vascular diseases.

Many studies have shown that OSAS is closely related to the risk factors of vascular diseases, such as hypertension, arrhythmia, CAD, stroke and pulmonary arterial hypertension (5-8). The morbidity and mortality rates of CAD in OSAS patients are significantly increased, but the exact relationship between OSAS and CAD remains unclear. In the present study, 84 patients with suspected CAD were enrolled for sleep detection and coronary angiography, in order to investigate the correlation between OSAS and CAD and analyze the risk factors.

\section{Materials and methods}

Subjects. A total of 84 patients with suspected CAD due to chest tightness, chest pain and nocturnal angina, admitted in the Department of Cardiology of The Affiliated Hospital of Medical College Qingdao University (Qingdao, China) from March, 2016 to June, 2017 were selected, including 
Table I. General conditions in different groups.

\begin{tabular}{lccr}
\hline & \multicolumn{2}{c}{ Groups } & \\
\cline { 2 - 4 } Items & Control $(\mathrm{n}=34)$ & CAD $(\mathrm{n}=50)$ & P-value \\
\hline Age (years) & $60.37 \pm 8.45$ & $69.96 \pm 7.48$ & 0.064 \\
Sex $(\mathrm{male} / \mathrm{female})$ & $16 / 18$ & $40 / 10$ & 0.071 \\
BMI $\left(\mathrm{kg} / \mathrm{m}^{2}\right)$ & $27.08 \pm 4.72$ & $25.34 \pm 4.03$ & 0.052 \\
LVEF $(\%)^{\mathrm{b}}$ & $70.26 \pm 6.98$ & $62.95 \pm 9.14$ & 0.004 \\
AHI $\left(\mathrm{n} / \mathrm{min}^{\mathrm{a}}\right.$ & $14.03 \pm 11.21$ & $21.22 \pm 13.66$ & 0.019 \\
Lowest oxygen saturation $^{\mathrm{a}}$ & $88.45 \pm 5.19$ & $84.32 \pm 7.59$ & 0.023 \\
TC $(\mathrm{mmol} / \mathrm{l})$ & $4.50 \pm 0.92$ & $4.12 \pm 0.89$ & 0.075 \\
TG $(\mathrm{mmol} /)^{\mathrm{a}}$ & $1.97 \pm 1.14$ & $2.33 \pm 1.78$ & 0.044 \\
Gensini score & $7.25 \pm 12.62$ & $42.37 \pm 38.81$ & 0.008 \\
Diabetes mellitus & $3(14.82 \%)$ & $39(78.00 \%)$ & 0.039 \\
Hypertension & $13(38.24 \%)$ & $29(58.00 \%)$ & 0.087 \\
Smoking & $3(8.82 \%)$ & $26(52.00 \%)$ & 0.003 \\
Obesity & $21(61.76 \%)$ & $40(80.00 \%)$ & 0.068 \\
OSAS & $21(61.76 \%)$ & & 0.056 \\
\hline
\end{tabular}

BMI, body mass index; LVEF, left ventricular ejection fraction; AHI, apnea hyponea index; TC, total cholesterol; TG, triglyceride; OSAS, obstructive sleep apnea syndrome; ${ }^{\mathrm{a}} \mathrm{P}<0.05$ in comparison with the control group; ${ }^{\mathrm{b}} \mathrm{P}<0.01$ in comparison with the control group.

56 males and 28 females. According to the results of coronary angiography, the patients were divided into two groups; the control group with a negative coronary angiography and the CAD group with a positive coronary angiography (Table I). Inclusion criteria were: i) Patients with repeated snoring for $>5$ years; ii) with chest tightness, chest pain or nocturnal angina, or with ST-segment depression or pathological Q wave in electrocardiogram; and iii) age between 18-75 years. Exclusion criteria were: i) Cardiac dysfunction or heart failure (ejection fraction $<40 \%$ ); ii) chronic obstructive pulmonary disease and daytime hypoxemia; iii) recent oxygen therapy or mechanical ventilation treatment; iv) valvular disease or cardiomyopathy; v) variant angina pectoris or X syndrome; vi) recent cerebral stroke; vii) central sleep apnea; and viii) intolerance to PSG monitoring. The present study was approved by the Ethics Committee of the Affiliated Hospital of The Affiliated Hospital of Medical College Qingdao University. Written informed consent was obtained from all the participants before the study.

\section{Methods}

General data collection. At admission, the patients were asked about their medical history in detail and received a questionnaire (habitual snoring in the questionnaire was defined as the snoring affecting the bedfellows for at least 3 nights a week). The vital signs were measured and the body mass index (BMI) was calculated. After fasting for $8 \mathrm{~h}$, blood was drawn for blood biochemistry, blood routine examination, myocardium and enzymogram. Before coronary angiography, echocardiography and electrocardiographic examination were performed.

Sleep monitoring. Before and after coronary angiography, the sleep respiratory monitoring was performed using the
Stardust II portable sleep monitor (Wellkang, Melbourne, FL, USA). Sleeping pills and drinking alcohol were prohibited at $48 \mathrm{~h}$ before sleep monitoring. The nose and mouth airflow, chest and abdomen movement, blood oxygen saturation in finger tips, heart rate, snoring, body position and other parameters of patients were monitored (4). Diagnostic criteria of OSAS: Recurrent episodes of apnea (nose and mouth breathing stops for $>10 \mathrm{sec}$ ) and hypopnea (respiratory airflow is reduced to $50 \%$ of normal airflow intensity, accompanied by decreased blood oxygen saturation of $>4 \%$ ) $>30$ times, or apnea hypopnea index $(\mathrm{AHI}) \geq 5$ recorded by the sleep monitor.

Coronary angiography. According to the guidelines of coronary angiography of American College of Cardiology (ACC)/American Heart Association (AHA), three experienced chief physicians performed the coronary angiography. The multi-location angiography was conducted using Judkin's method. The image processing system of angiography machine was used for the quantitative analysis of stenosis. Diagnostic criteria of coronary angiography: Stenosis of at least one coronary artery or its major lumen $>50 \%$ indicated the positive results (Fig. 1).

Gensini score. According to the results of coronary angiography, the degree of vascular lesion was quantitatively scored using the Gensini scoring system. The scoring criteria were composed of two parts: i) The basic score was given according to the degree of coronary artery stenosis; and ii) the scoring coefficient was determined according to the location of coronary artery lesions. The accumulated total in each lesion site was the total score of severity of coronary artery lesions in patients. 

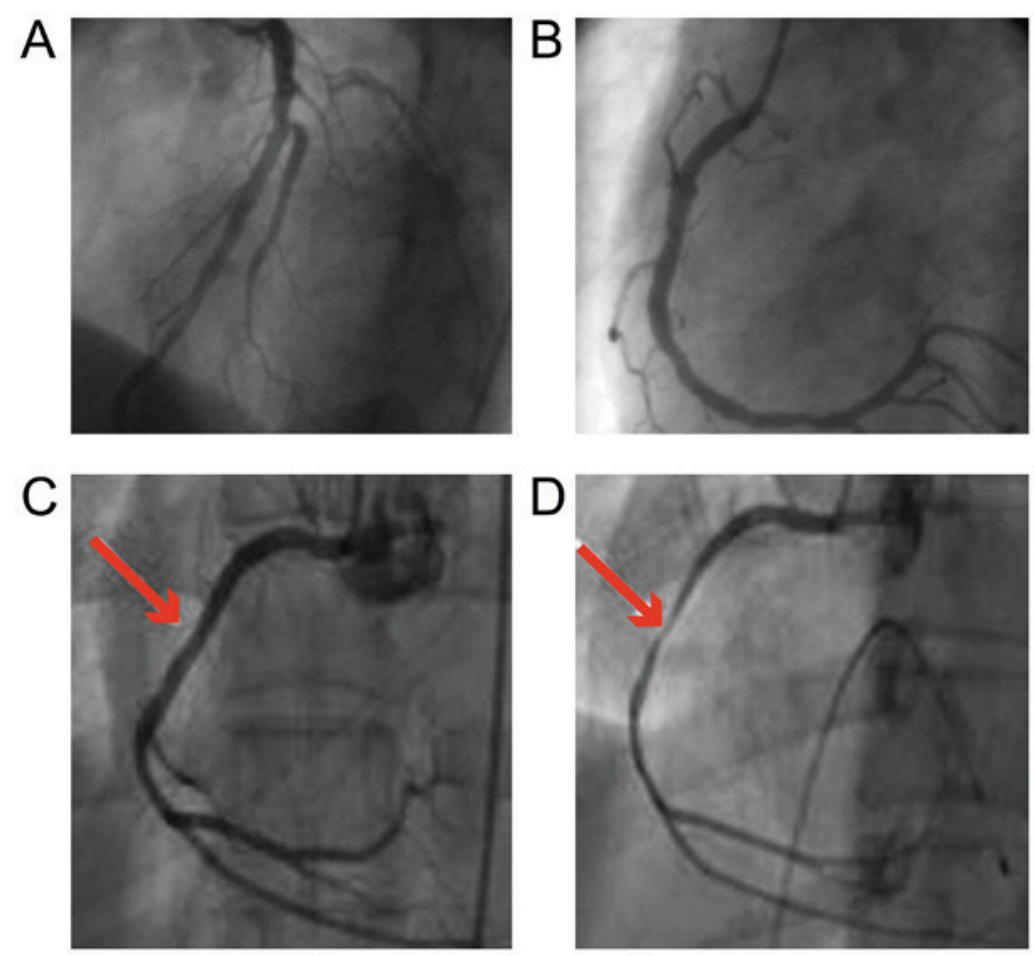

Figure 1. Coronary angiography negative (A-B) and positive images (C-D). (A) The left coronary artery (LCA). (B) The right coronary artery (RCA). (C) Insignificant stenosis is shown at corresponding segment (red arrow) on coronary angiography. (D) Provoked severe stenosis is revealed at same site (red arrow) on coronary angiography after intracoronary administration of ergonovine.

Table II. Relationship between coronary artery involvement and Gensini score and severity of OSAS in CAD group.

\begin{tabular}{|c|c|c|c|c|c|c|}
\hline \multirow[b]{2}{*}{ CAD group } & \multicolumn{4}{|c|}{ Coronary artery involvement } & \multirow[b]{2}{*}{ Gensini } & \multirow[b]{2}{*}{ P-value } \\
\hline & 0 & 1 & 2 & 3 & & \\
\hline Complicated with mild OSAS & 0 & 7 & 0 & 6 & $19.38 \pm 20.15^{\mathrm{a}}$ & 0.045 \\
\hline Complicated with moderate OSAS & 0 & 4 & 2 & 6 & $25.42 \pm 19.61^{\mathrm{a}}$ & 0.037 \\
\hline Complicated with severe OSAS & 2 & 9 & 3 & 11 & $60.16 \pm 43.39^{\mathrm{a}}$ & 0.028 \\
\hline
\end{tabular}

${ }^{\mathrm{a}} \mathrm{P}<0.05$; CAD, coronary artery disease; OSAS, obstructive sleep apnea syndrome.

Statistical analysis. All statistics were performed using SPSS 20.0 software, (IBM, Armonk, NY USA). Comparison between groups was done using One-way ANOVA test followed by post hoc test (least significant difference). Percentage (\%) was used to express the enumeration data and Chi-square test was used for data analysis. One-way analysis of variance was used to evaluate the relationship between relative risk factors and CAD. The statistically significant factors were screened for multivariate logistic regression analysis. $\mathrm{P}<0.05$ was considered to indicate a statistically significant difference.

\section{Results}

General conditions. There were statistically significant differences in AHI, lowest oxygen saturation, left ventricular ejection fraction, triglyceride level, Gensini score, smoking and diabetes mellitus between the CAD and control groups
$(\mathrm{P}<0.05)$. BMI, total cholesterol level, OSAS, hypertension, hyperlipidemia, obesity and age were not statistically significant between the two groups $(\mathrm{P}>0.05)$. The more severe OSAS was identified in the CAD group compared to that in the control group, but the statistical analysis showed that there was no statistically significant difference between the two groups (80.00 vs. 61.76\%, $\mathrm{P}=0.056)$ (Table I).

Correlation analysis of $C A D$ and OSAS. The cumulative number of vessels in CAD group was high, and the coronary artery stenosis of moderate-severe patients generally involved multiple vessels. The CAD patients were divided into mild, moderate and severe group according to the severity of OSAS. The difference in Gensini score was significant among the three groups, and the analysis of variance showed the statistically significant difference $(\mathrm{P}=0.025)$. The more severe the OSAS was, the higher the Gensini score would be, suggesting that OSAS is closely related to CAD (Table II). 
Table III. Univariate analysis of CAD-related risk factors.

\begin{tabular}{|c|c|c|c|c|}
\hline Risk factor & No. & CAD n $(\%)$ & $\chi^{2}$ & P-value \\
\hline Smoking $^{\mathrm{b}}$ & 32 & $29(90.63 \%)$ & 10.375 & $<0.001$ \\
\hline Hyperlipidemia $^{a}$ & 40 & $30(75.00 \%)$ & 6.934 & 0.034 \\
\hline Obesity & 47 & $26(55.32 \%)$ & 1.358 & 0.528 \\
\hline Hypertension $^{\mathrm{b}}$ & 52 & $39(75.00 \%)$ & 7.116 & 0.006 \\
\hline Diabetes mellitus & 10 & $6(60.00 \%)$ & 2.217 & 0.615 \\
\hline $\mathrm{AHI}>20^{\mathrm{a}}$ & 34 & $27(79.41 \%)$ & 5.623 & 0.027 \\
\hline
\end{tabular}

${ }^{\mathrm{a}} \mathrm{P}<0.05 ;{ }^{\mathrm{b}} \mathrm{P}<0.01 ; \mathrm{CAD}$, coronary artery disease; AHI, apnea hypopnea index.

Table IV. Multivariate logistic analysis of CAD-related risk factors.

\begin{tabular}{lccc}
\hline Risk factor & OR & Wald & P-value \\
\hline Hypertension & 3.489 & 2.126 & 0.087 \\
Smoking $^{\mathrm{a}}$ & 7.036 & 5.204 & 0.029 \\
Hyperlipidemia & 3.125 & 1.469 & 0.146 \\
AHI $>20^{\mathrm{a}}$ & 5.377 & 2.478 & 0.038 \\
\hline
\end{tabular}

${ }^{\mathrm{a}} \mathrm{P}<0.05$; CAD, coronary artery disease; $\mathrm{AHI}$, apnea hypopnea index.

Univariate analysis of CAD-related risk factors. Univariate analysis was performed for screening with CAD as the dependent variable and the risk factors as the independent variables. The results showed that the hypertension $(\mathrm{P}=0.006)$, hyperlipidemia $(\mathrm{P}=0.034)$, smoking $(\mathrm{P}<0.001)$ and $\mathrm{AHI}>20$ $(\mathrm{P}=0.027)$ had significant correlations with $\mathrm{CAD}$, but $\mathrm{AHI}>5$ had no correlation with CAD (P>0.05) (Table III).

Multivariate logistic analysis of CAD-related risk factors. The risk factors obtained via univariate analysis were included into the multivariate logistic regression, and the results showed that smoking $(\mathrm{P}=0.029)$ and $\mathrm{AHI}>20(\mathrm{P}=0.038)$ were the risk factors of $\mathrm{CAD}$, and they had significantly positive correlations with $\mathrm{CAD}$, odds ratio $(\mathrm{OR})=7.036$ and 5.377 Table IV).

\section{Discussion}

SAS is a common disease, clinically dominated by OSAS, which can exist in a variety of cardiovascular system diseases, such as congestive heart failure, hypertension and CAD (1-3). Sleep structural disorder and hypoxemia in sleep stage are important features of OSAS. The most common cardiovascular symptoms of OSAS are stable angina and myocardial infarction. The incidence of angina pectoris and myocardial infarction in patients with OSAS and CAD is higher and more pronounced.

The results of this study showed that the lowest oxygen saturation was significantly different in CAD and control group $(\mathrm{P}=0.023)$. AHI was higher but the lowest oxygen saturation was lower in CAD group $(\mathrm{P}<0.05)$. Furthermore, CAD group was divided into mild, moderate and severe group according to the severity of OSAS. The Gensini score had statistically significant difference among the three groups, and it was increased with the increasing severity of OSAS $(\mathrm{P}<0.05)$. Univariate analysis suggested that the hypertension $(\mathrm{P}=0.006)$, hyperlipidemia $(\mathrm{P}=0.034)$, smoking $(\mathrm{P}<0.001)$ and AHI $>20(\mathrm{P}=0.027)$ had significant correlations with CAD, but $\mathrm{AHI}>5$ had no correlation with $\mathrm{CAD}(\mathrm{P}>0.05)$. Logistic regression analysis was performed for the CAD-related risk factors. The results showed that $\mathrm{AHI}>20$ was one of the risk factors of $\mathrm{CAD}(\mathrm{P}=0.038)$, which was consistent with the literature in China and world-wide $(9,10)$. It has been reported that OSAS, like obesity, smoking and hypertension, is an independent risk factor of myocardial infarction $(2,3,8,11)$. The findings of American Sleep Health Research Center also showed that AHI $>5$ is a risk factor of cardiovascular disease and can significantly increase the incidence and mortality rates of cardiovascular events. Studies have also pointed out that OSAS is also an important factor affecting the prognosis of CAD (12). This study showed that the moderate-severe OSAS is one of the risk factors of CAD, and the probability of CAD in moderate-severe OSAS patients is 5,377 times that in non-OSAS patients.

The main influencing mechanisms of OSAS (10,13-16), as a risk factor of CAD, are as follows: i) Hypoxemia in OSAS patients caused by repeated apnea can activate the chemical receptors and sympathetic nerve, resulting in vascular smooth muscle remodeling and hypertrophy, increasing oxygen consumption of heart and aggravating myocardial ischemia and hypoxia; ii) the platelet activity and aggregation capacity in OSAS patients are increased, leading to the occurrence of cardiovascular events; iii) the activity and content of tissue plasminogen activator inhibitor in OSAS patients are increased, inhibiting the fibrinolytic system in the body and leading to the hypercoagulable state, thrombosis and atherosclerosis development; iv) repeated apnea hypoxia activates the oxidative stress, which further leads to the lipid peroxidation, vascular endothelial cell injury and increased aggregation of inflammatory factors; v) nocturnal hypoxia may affect the activity of lipoprotein receptor, leading to the increased blood lipids and aggravating the development of atherosclerosis; and vi) accompanying factors: OSAS patients are mostly complicated by obesity, lipid metabolism disorders, hypertension, arrhythmia and other susceptibility factors of CAD. In order to reduce the incidence rate and improve the prognosis of CAD in OSAS patients, therefore, it is 
recommended to provide appropriate prevention and treatment for OSAS, as a risk factor.

The multivariate logistic regression analysis showed that smoking was also positively correlated with CAD, and was one of the risk factors of CAD (correlation coefficient $=7.036$, $\mathrm{P}<0.001)$. Epidemiological investigation and clinical studies worldwide have also confirmed that smoking is an independent risk factor of CAD $(11,17,18)$. The risk of CAD for smokers is 1.5 - to 4 -fold that of non-smokers. Smoking can cause cardiovascular events including possible mechanism leading to changes in hematology, neurohumor, metabolism, hemodynamics, molecular genetics and biochemistry, causing coronary artery spasm or atherosclerosis, eventually resulting in CAD. Therefore, smoking cessation is an important measure to reduce the risk of CAD.

Logistic regression analysis revealed that hypertension and hyperlipidemia were not significant risk factors for coronary heart disease $(\mathrm{P}=0.087)$, which may be associated with the limited experimental cases.

CAD is one of the important causes of human death, which has been confirmed to be related to age, sex, blood lipids, blood pressure, diabetes mellitus, obesity and smoking. Nevertheless, there is little research on the correlation between OSAS and CAD. The results of the present study have shown that $\mathrm{AHI}>20$ was a risk factor of $\mathrm{CAD}(\mathrm{P}=0.038)$, and the Gensini score of CAD is related to the severity of OSAS. It is recommended that CAD patients receive PSG monitoring for diagnosis and treatment in time. In order to improve the prognosis of CAD patients, OSAS, as a risk factor, should be actively prevented and treated. The timely diagnosis and treatment of OSAS in CAD patients are secondary preventive measures of CAD. However, the small sample size is a limitation of the present study, and we would like to increase the sample size in future studies.

In conclusion, the results of the present study have shown that CAD is closely correlated with OSAS. In addition, smoking and $\mathrm{AHI}>20$ are the risk factors of CAD, which should be paid close attention. To reduce the incidence rate and improve the prognosis of CAD, patients should quit smoking and OSAS, as a risk factor, should be prevented and treated appropriately.

\section{Acknowledgements}

Not applicable.

\section{Funding}

Not funding was received.

\section{Availability of data and materials}

All data generated or analyzed during this study are included in this published article.

\section{Authors' contributions}

JZ and WZ designed the study, YS performed the collection and the entry of the data, YJ analysed the data, JZ prepared the manuscript, YY carried out the search and the literature analysis. All the authors read and approved the final manuscript.

\section{Ethics approval and consent to participate}

This study was approved by the Ethics Committee of The Affiliated Hospital of Medical College Qingdao University (Qingdao, China). Signed written informed consents were obtained from the patients and/or guardians.

\section{Consent for publication}

Not applicable.

\section{Competing interests}

The authors declare that they have no competing interests.

\section{References}

1. Imataka G, Nakajima I, Goto K, Konno W, Hirabayashi H and Arisaka O: Drop episodes improved after tracheotomy: A case of Coffin-Lowry syndrome associated with obstructive sleep apnea syndrome. Eur Rev Med Pharmacol Sci 20: 498-501, 2016.

2. Lombardi C, Tobaldini E, Montano N, Losurdo A and Parati G: Obstructive sleep apnea syndrome (OSAS) and cardiovascular system. Med Lav 108: 276-282, 2017.

3. Barbosa FR, Silva CM, Lima GA, Warszawski L, Domingues RC, Dominic M, Fontes R, Vieira Neto L and Gadelha MR: Prevalence of obstructive sleep apnea in patients with prolactinoma before and after treatment with dopamine agonists. Pituitary 17: 441-449, 2014.

4. Buccheri A, Chinè F, Fratto G and Manzon L: Rapid maxillary expansion in obstructive sleep apnea in young patients: Cardio-Respiratory monitoring. J Clin Pediatr Dent 41: 312-316, 2017.

5. Karimzadeh F, Nami M and Boostani R: Sleep microstructure dynamics and neurocognitive performance in obstructive sleep apnea syndrome patients. J Integr Neurosci 16: 127-142, 2017.

6. Alvarez-Sabín J, Romero O, Delgado P, Quintana M, Santamarina E, Ferré A, Maisterra O, Riba-Llena I, Montaner J and Sampol G: Obstructive sleep apnea and silent cerebral infarction in hypertensive individuals. J Sleep Res 20: 962-1105, 2017.

7. Li L, Lu J, Xue W, Wang L, Zhai Y, Fan Z, Wu G, Fan F, Li J, Zhang C, et al: Target of obstructive sleep apnea syndrome merge lung cancer: Based on big data platform. Oncotarget 8: 21567-21578, 2017

8. Micarelli A, Liguori C, Viziano A, Izzi F, Placidi F and Alessandrini $M$ : Integrating postural and vestibular dimensions to depict impairment in moderate-to-severe obstructive sleep apnea syndrome patients. J Sleep Res 26: 487-494, 2017.

9. Arik B, Inci MF, Gumus C, Varol K, Ege MR, Dogan OT and Zorlu A: Advanced age and apnea-hypopnea index predict subclinical atherosclerosis in patients with obstructive sleep apnea syndrome. Multidiscip Respir Med 8: 9, 2013.

10. Meng S, Fang L, Wang CQ, Wang LS, Chen MT and Huang XH: Impact of obstructive sleep apnoea on clinical characteristics and outcomes in patients with acute coronary syndrome following percutaneous coronary intervention. J Int Med Res 37: 1343-1353, 2009.

11. Zhu H, Xu H, Chen R, Liu S, Xia Y, Fu Y, Li X, Qian Y, Zou J, Yi H, et al: Smoking, obstructive sleep apnea syndrome and their combined effects on metabolic parameters: Evidence from a large cross-sectional study. Sci Rep 7: 8851, 2017.

12. Capodanno D, Milazzo G, Cumbo M, Marchese A, Salemi A, Quartarone L, Benvenuto E, Galseran C, Distefano SM and Tamburino C: Positive airway pressure in patients with coronary artery disease and obstructive sleep apnea syndrome. J Cardiovasc Med (Hagerstown) 15: 402-406, 2014.

13. Singh A, Prasad R, Garg R, Kant S, Hosmane GB, Dubey A, Agarwal A and Verma RK: A study to estimate prevalence and risk factors of obstructive sleep apnoea syndrome in a semi-urban Indian population. Monaldi Arch Chest Dis 87: 773, 2017.

14. Mihaicuta S, Udrescu M, Topirceanu A and Udrescu L: Network science meets respiratory medicine for OSAS phenotyping and severity prediction. PeerJ 5: e3289, 2017. 
15. Wang ZH, Zhu D, Xie S, Deng Y, Pan Y, Ren J and Liu HG: Inhibition of rho-kinase attenuates left ventricular remodeling caused by chronic intermittent hypoxia in rats via suppressing myocardial inflammation and apoptosis. J Cardiovasc Pharmacol 70: 102-109, 2017.

16. Vitulano N, Di Marco Berardino A, Re A, Riccioni G, Perna F Mormile F, Valente S and Bellocci F: Obstructive sleep apnea and heart disease: The biomarkers point of view. Front Biosci (Schol Ed) 5: 588-599, 2013.

17. Foody J, Huo Y, Ji L, Zhao D, Boyd D, Meng HJ, Shiff S and Hu D: Unique and varied contributions of traditional CVD risk factors: A systematic literature review of CAD risk factors in China. Clin Med Insights Cardiol 7: 59-86, 2013.
18. Schmermund A, Lehmann N, Bielak LF, Yu P, Sheedy PF II, Cassidy-Bushrow AE, Turner ST, Moebus S, Möhlenkamp S, Stang A, et al: Comparison of subclinical coronary atherosclerosis and risk factors in unselected populations in Germany and USA. Atherosclerosis 195: e207-e216, 2007.

(i) (9) This work is licensed under a Creative Common

(c) $\mathrm{EY}$ No ND Attribution-NonCommercial-NoDerivatives 4.0 International (CC BY-NC-ND 4.0) License. 